\title{
HACIA UN MODELO DE VALUACIÓN DE ACTIVOS DE CAPITAL PARA MÉXICO: ANÁLISIS DE ACTIVOS INDIVIDUALES CON COEFICIENTES VARIANTES EN EL TIEMPO
}

\author{
Towards a Model of Capital Asset Pricing \\ FOR MeXico: ANALYSIS INDIVIDUAL ASSETS \\ TIME-VARYING COEFFICIENTS \\ Francisco López-Herrera* \\ Humberto Valencia-Herrera**
}

(Recibido: octubre, 2015/Aceptado: febrero, 2016)

\section{Resumen}

Analizando el comportamiento de los coeficientes beta variantes en el tiempo, estimados mediante el filtro de Kalman para una muestra de activos mexicanos que cotizan en la bolsa mexicana de valores, se observa que un modelo con betas variantes en el cual los rendimientos de los activos dependen de un factor local y uno internacional permiten estimar las primas de riesgo de estos activos. Dado la dependencia que exhiben todos los activos analizados en relación con un factor doméstico, la evidencia que se muestra en este trabajo da soporte a la hipótesis de que el mercado mexicano no está integrado completamente al internacional. Es decir, en nuestro análisis se encuentra que las características del riesgo sistemático de los activos mexicanos estudiados son congruentes con la valuación de activos en un contexto de mercado parcialmente segmentado. Como consecuencia, los

\footnotetext{
* División de Investigación, Facultad de Contaduría y Administración, UNAM, México. Correo electrónico: francisco_lopez_herrera@yahoo.com.mx.

** Profesor-investigador del Tecnológico de Monterrey, México, humberto. Correo electrónico: valencia@ itesm.mx.
} 
resultados aquí mostrados sugieren que es adecuado el uso de un modelo internacional de valuación de activos (ICAPM), con un factor internacional y un factor doméstico, y coeficientes beta variantes en el tiempo en lugar de modelos con un solo factor nacional.

Palabras clave: ICAPM, beta variante en el tiempo, integración de mercados, filtro de Kalman.

Clasificación JEL: G15, G12.

\section{Abstract}

Analyzing the behavior of time-variyng beta coefficients estimated through the Kalman filter for a sample of Mexican assets listed on the Mexican Stock Exchange, it can be seen that a model with changing betas, in which asset returns depend on a local factor and an international one, allows to estimate risk premiums of these assets. Given the dependence exhibited all the studied assets in relation to a domestic factor, the evidence shown in this study supports the hypothesis that the Mexican market is not fully integrated into the international. That is, in our analysis is found that the characteristics of the systematic risk of the Mexican studied assets are consistent with the valuation of assets in the context of partially segmented market. Consequently, the results shown here suggest that it is appropriate to use a international model of asset valuation (ICAPM), with an international factor and a domestic factor, and time-varying beta coefficients instead of models with a single domestic factor.

Keywords: ICAPM, time-varying beta, markets integration, Kalman Filter. JEL classification: G15, G12.

\section{Introducción}

La teoría de selección de los portafolios riesgosos postulada por Markowitz (1952), constituye la base teórica del Modelo de Valuación de Activos de Capital (CAPM), desarrollado de manera independiente por Sharpe (1963, 1964), Lintner (1965), Mossin (1966) y Treynor (1961, 1962). El CAPM, de 
carácter positivo, a diferencia del carácter normativo del modelo de Markowitz, constituye el primer intento de formulación rigurosa de una teoría del mercado de capitales, la cual, según Sharpe $(1970,2000)$ busca predecir las relaciones entre las variables importantes en el equilibrio:

“...una situación de equilibrio, una vez alcanzada, se mantendrá. En desequilibrio hay presiones para el cambio, en equilibrio no hay alguna. ...El cambio en el mercado de capitales toma forma de alteraciones en las tenencias de los títulos [por parte de los inversionistas] y sus precios. Los precios de equilibrio (y las tenencias) son consistentes con la riqueza, preferencia y predicciones de las personas...En un sentido más amplio, los precios de equilibrio son determinados por la riqueza, preferencias y predicciones de las personas."

De acuerdo con Sharpe, Gordon y Bailey (1999, p. 228) el CAPM se fundamenta en los siguientes supuestos:

1. Los inversionistas evalúan los portafolios observando los rendimientos esperados y las desviaciones estándar para un periodo.

2. Los inversionistas nunca están satisfechos; así, ante una elección entre dos portafolios idénticos en las otras características, escogerán el que tiene el mayor rendimiento esperado.

3. Los inversionistas son aversos al riesgo, por ello ante una elección entre dos portafolios iguales en todo lo demás, escogerán el que tiene menor desviación estándar. ${ }^{1}$

4. Los activos individuales son infinitamente divisibles, esto significa que un inversionista puede comprar una fracción de una acción si así lo decide.

5. Hay una tasa libre de riesgo a la que el inversionista puede prestar (invertir) o pedir prestado.

6. Los costos de los impuestos y de las transacciones son irrelevantes.

7. Todos los inversionistas tienen el mismo horizonte temporal de inversión (un periodo).

8. La tasa libre de riesgo es la misma para todos los inversionistas.

\footnotetext{
${ }^{1}$ Aunque en el criterio original de Markowitz se propone la varianza (dispersión) de los rendimientos para medir el riesgo, no existe problema alguno al utilizar la desviación estándar para tal propósito ya que la inferencia respectiva se mantiene.
} 
9. La información está disponible para todos los inversionistas de manera gratuita e instantánea.

10. Los inversionistas tienen expectativas homogéneas. Esto significa que tienen las mismas percepciones en relación con los rendimientos esperados, desviaciones estándar y covarianzas de los valores.

El CAPM ofrece una explicación sobre la relación entre el rendimiento esperado y el riesgo de los activos y de los portafolios, al mismo tiempo que establece una medida para el riesgo (Sharpe, 1970, 2000). El punto de partida es la existencia de un hipotético portafolio del mercado, $M$, compuesto por todos los activos riesgosos disponibles en el mercado y cuyo rendimiento esperado es $E_{M^{\prime}}$ con riesgo $\sigma_{M}$. De acuerdo con el teorema de separación de Tobin, $M$ es el único portafolio de activos riesgosos que maximiza la recompensa por el riesgo asumido, $\left(E_{M}-r_{f}\right) / \sigma_{M}$, en virtud de que todos los inversionistas están de acuerdo en que $M$ es el portafolio óptimo de activos riesgosos. Siendo el conjunto de activos con riesgo el mismo conjunto de oportunidades para todos los inversionistas, las diferencias en la cantidad del portafolio de activos riesgosos que tienen los inversionistas en su poder se deben sólo a las diferentes preferencias por el riesgo y el rendimiento.

Por lo anterior, en el equilibrio, los inversionistas tendrán en su portafolio la misma combinación de activos con riesgo, con las mismas proporciones relativas y complementarán su portafolio invirtiendo en el activo libre de riesgo o endeudándose a la tasa libre de riesgo para invertir en el portafolio de activos riesgosos (teorema de separación Tobin), de tal forma que alcancen la combinación de riesgo y rendimiento preferida. Como resultado, la combinación óptima de activos con riesgo, en el sentido de la eficiencia Markowitz, puede determinarse sin conocimiento alguno de las preferencias del inversionista respecto al riesgo y al rendimiento.

Grubel (1968) extendió la teoría de selección del portafolio al contexto de la inversión internacional, contribuyendo con ello a que el análisis del riesgo de los portafolios también se ocupara del riesgo al que están sujetos los portafolios de activos riesgosos de diferentes países. Evidentemente esto es relevante para los inversionistas que buscan los beneficios de la diversificación internacional de portafolios, manteniendo para ello tenencias de activos de varios países en sus portafolios, tanto individuales como institucionales, así como para los administradores de esos portafolios.

Naturalmente, la globalización de los mercados financieros ha permitido que la inversión internacional de portafolio se haya vuelto cada vez más 
común. La importancia que tiene lograr un mejor entendimiento de la naturaleza y características del riesgo de ese tipo de inversiones es grande y no sólo de orden práctico, pues también desde la perspectiva meramente académica se vuelve relevante entender mejor esos riesgos para obtener mejores explicaciones económicas de las relaciones entre el rendimiento de esos activos y el riesgo al que están expuestos. El tema no es sólo relevante desde la perspectiva de las inversiones internacionales de portafolio, dado que la relación entre el rendimiento y el riesgo es uno de los determinantes del costo de capital, también alcanza su relevancia al tema de las inversiones en proyectos productivos por parte de las empresas de diferentes sectores de actividad económica.

Desde los estudios pioneros sobre un CAPM internacional, los de Solnik (1974), Sercu (1980), y Adler y Dumas (1983), por ejemplo, ha quedado claro que la relación que el rendimiento y riesgo deben tener en el equilibrio es más compleja que la del CAPM en su versión doméstica. Desde el punto de vista práctico, además de los problemas típicos derivados de las relaciones entre los tipos de cambio involucrados, se encuentran otros aspectos como las cargas impositivas y las regulaciones de los mercados domésticos. En la medida que la globalización e internacionalización de los mercados de capitales avanzan, algunos de estos problemas se vuelven menos importantes al ser retirados mediante los cambios regulatorios y la liberalización.

La integración de los mercados hace entonces que las características del riesgo sistemático de los activos sean diferentes en comparación con el riesgo sistemático en un contexto de segmentación de los mercados. De acuerdo con Alford (1993), la inversión en activos riesgosos internacionales enfrenta esencialmente tres tipos de riesgos: $a$ ) el riesgo no sistemático de cada activo individual, naturalmente susceptible de diversificarse en forma total mediante la diversificación del portafolio; $b$ ) un riesgo relacionado con el país de los activos o riesgo sistemático local que únicamente puede diversificarse mediante la tenencia de un portafolio que incluya activos internacionales; y c) el riesgo sistemático relacionado con la economía mundial, y que no se puede diversificar.

Errunza y Losq (1985) muestran que los mercados de capital en el mundo se pueden encontrar en un espectro que va desde la segmentación perfecta hasta la integración perfecta. En los mercados perfectamente segmentados la valoración de los activos se da exclusivamente sobre una base nacional, sin que interfieran en forma alguna las fuerzas internacionales, pues existen suficientes barreras para que los inversionistas locales no puedan adquirir 
activos foráneos ni los inversionistas extranjeros puedan comprar activos domésticos que les resulten rentables.

En el extremo opuesto, la integración perfecta implica que los precios de los activos se establecen mediante una base netamente internacional. Entre los extremos, se encuentran los mercados parcialmente segmentados, en los que los factores nacionales que determinan los precios de los activos son importantes, pero en la fijación de los precios influyen parcialmente también factores de carácter internacional.

Por otra parte es conveniente destacar que existe una amplia literatura que justifica el uso de coeficientes beta variantes en el tiempo, particularmente para activos individuales, razón por la cual, utilizando las propiedades de series de tiempo de los coeficientes beta variantes en el tiempo, este artículo examina la relación de las betas de los activos con mayor tiempo de cotización en la bolsa mexicana de valores (BMV) mediante un análisis de series temporales que utiliza la técnica de filtro de Kalman. El artículo mejora la descripción de las conductas de los rendimientos de los activos en la BMV analizando efectos locales y globales, al permitir betas variables en el tiempo (Longstaff 1989), brindando elementos que justifican el uso de modelos como el modelo condicional internacional de valuación de activos de capital, ICAPM, por sus siglas en inglés (International Capital Asset Pricing Model) para explicar los rendimientos en la BMV.

En el contexto del modelo condicional ICAPM, el estudio da elementos para evaluar el grado de eficiencia en el sentido de Fama con que funciona el mercado mexicano. Para la mayoría de los activos las alfas encontradas son negativas o no significativas, en el primer caso se puede pensar en que existe una especie de ajuste que elimina primas de riesgo no explicadas por los dos factores de riesgo sistemático incluidos en el modelo (doméstico e internacional), en tanto que en el segundo caso se deduce que ambos factores son suficientes para explicar los rendimientos de los activos. El resto del artículo está organizado en las siguientes secciones: revisión de literatura, aspectos metodológicos, los resultados empíricos y las conclusiones.

\section{Revisión de literatura}

Existe una amplia literatura que justifica el uso de coeficientes beta variantes en el tiempo, particularmente para activos individuales. Blume (1971) publicó 
el primer estudio relacionado con la estabilidad de la beta. Usando periodos de siete años y los rendimientos mensuales de 1926-1968, encontró que las betas históricas de los valores individuales y los portafolios más pequeños son de utilidad, pero limitada, para pronosticar el valor de las próximas betas. También señaló que los coeficientes betas históricos para los portafolios más grandes son notablemente precisos en la predicción de futuras betas de un portafolio.

Levy (1971) encontró que la evaluación de los riesgos futuros es muy fiable para grandes portafolios, algo menos fiable para los portafolios más pequeños y muy poco fiable para valores individuales. También encontró que la previsibilidad mejora materialmente cuando el periodo del pronóstico se alarga, con una mayor mejoría al pasar de 13 semanas a 26 semanas, que cuando se pasa de 26 semanas a 52 semanas. En resumen, concluyó que para los portafolios de 25 acciones y más grandes, en los intervalos de predicción de 26 semanas o más, los coeficientes beta últimos son excelentes sustitutos de futuros coeficientes beta.

Meyers (1973) presenta evidencia adicional de la estabilidad de las betas de valores individuales utilizando intervalos de un mes de diferencia y periodos de tiempo de por lo menos de siete años a partir de agosto 1952 hasta diciembre de 1967. Se encontró evidencia indiscutible de graves violaciones al supuesto de estabilidad. Levitz (1974) llegó a la conclusión de que los coeficientes beta del portafolio son muy estables, mientras que los coeficientes betas de valores individuales son muy inestables.

Baesel (1974) concluyó que la estabilidad de los coeficientes beta depende tanto del intervalo de estimación utilizado y de lo extremo que sea el coeficiente beta elegido. Trabajó con datos de Estados Unidos desde 1950 hasta 1967, señaló que las betas de los menores valores de riesgo y las de mayores riesgos mostraron una mayor estabilidad intertemporal. Una observación similar lo hace Sharpe y Cooper (1972).

Blume (1979) informó que los coeficientes beta de activos individuales muestran una tendencia de regresión (hacia 1.0), que representa inestabilidad en sus valores beta. Barry y Wickler (1976) proponen que la inestabilidad en la beta de activos individuales podría deberse a las características cambiantes de la empresa, como es la estructura de capital, las políticas de los negocios, la estrategia de marketing y el entorno económico en el que opera la empresa. 
Fabozzi y Francis (1978) mostraron que el beta puede ser un coeficiente aleatorio. Alexander y Chervany (1980) utilizaron la desviación absoluta media como una medida de la estabilidad y mostraron que las betas extremas son menos estables en comparación con las betas interiores. También contradicen a Baesel (1974) y afirman que los intervalos de estimación más largos producen betas más estables. De acuerdo a Alexander y Chervany (1980), el intervalo de estimación óptima es de cuatro a seis años. También mostraron que, independientemente de la forma en que los portafolios se formen, la inestabilidad beta disminuye a medida que aumenta el número de títulos del portafolio.

Bildersee y Roberts (1981) mostraron que durante los periodos de fluctuación de las tasas de interés, los coeficientes beta varían de forma sistemática. Goldberg y Vora (1981) presentan evidencia empírica de un modelo de reversión de la beta. Ellos mostraron que altas (bajas) betas tienen mayores (menores) estimados en los mercados alcistas que en los mercados bajistas. Hawawini (1983) presenta un modelo que explica la dirección y el tamaño de los cambios en beta resultantes de los cambios en el intervalo de muestreo. Informó que a medida que el intervalo se alarga, los coeficientes beta de valores con escaso volumen de negociación (un problema que es particularmente importante para las empresas más pequeñas) aumentan, mientras que los coeficientes beta de acciones muy negociadas (probablemente de grandes empresas) disminuyen.

Brailsford y Josev (1997) proporcionan evidencia empírica de la bolsa de valores de Australia que es consistente con esta predicción. Carroll y Sears (1994) encuentra que las betas cambian cuando hay un anuncio de pago de dividendos, y estos cambios son diferentes si las betas son grandes o pequeñas. Allen et al.(1994), trabajando con una muestra de cerca de 2500 títulos estadounidenses a nivel individual y a nivel de portafolio, concluyen que el coeficiente beta de un portafolio no es más estable o no menos estable que los coeficientes beta de valores individuales.

Choudhry (2002) investiga la estructura estocástica de los coeficientes beta variables en el tiempo de 15 empresas del Reino Unido, a través de un modelo MA-GARCH bivariado. Los resultados muestran que los coeficientes beta variables en el tiempo son reversibles, pero sólo unos pocos tienen una memoria larga y por lo tanto revierten a un ritmo lento. El estudio justifica la estacionariedad de los coeficientes beta, que implica que los rendimientos de las acciones se pueden pronosticar en el largo plazo. Deb y 
Mistra (2011) examinan la "estabilidad" de los coeficientes beta con el tiempo en el mercado de capital de la India, tanto a través de periodos de calendario como a través de algunos "periodos de control" o subperiodos, correspondientes a las fases alcistas y bajistas en el mercado. Encuentran evidencia de inestabilidad de betas en los horizontes de tiempo más cortos, la cual se reduce cuando el periodo de estimación aumenta. También observan que las betas extremas (tanto muy bajas y muy altas) exhiben mucha mayor estabilidad que la gama intermedia de betas. El análisis lo realizan a nivel de activos individuales y de portafolios, encontrando resultados similares.

Braun y Sunier (1995) introducen un modelo GARCH para betas. Shanken (1990) and Ferson y Harvey (1993) condicionan betas en instrumentos agregados y Avramov y Chordia (2006) usan instrumentos a nivel de empresa. Jostova y Philipov (2005) proponen un proceso estocástico de reversión a la media para los coeficientes beta del mercado. En un estudio de simulación, el modelo propuesto genera estimaciones de beta significativamente más precisas que las betas estimadas con el modelo $\mathrm{GARCH}$, betas condicionadas en las variables agregadas o a nivel de empresa. Su modelo ofrece soporte sustancialmente más fuerte para el ICAMP condicional en relación con otros modelos de beta y ayuda a resolver anomalías en los precios de activos como la razón de valor en libros a valor de mercado y efectos de volatilidad idiosincrásicos en estudios de corte transversal del rendimiento de las acciones.

Diferentes esfuerzos se han hecho para modelar coeficientes beta que sean variantes en el tiempo. Avramov y Chordia (2005) proponen betas escaladas por características como son el tamaño de la empresa o la relación de valor de mercado a valor en libros. Shanken (1990) propone condicionar las betas individuales a variables agregadas como el nivel y la volatilidad de las tasas de interés. Ledoit, Santa-Clara, y Wolf (2003) proponen modelar las betas con un modelo GARCH implícito.

En el mercado mexicano se ha hecho poco avance al respecto, López et al. (2012) estudian una muestra de acciones mexicanas y estiman la beta variante del CAPM en su versión doméstica, encontrando evidencia mediante el filtro de Kalman de que dicho parámetro del modelo es variante en el tiempo. En Ramírez-Cedillo et al. (2014) se ha mostrado evidencia adicional sobre la variabilidad del coeficiente beta doméstico valiéndose del filtro de Schlicht y Ludsteck (2006) como alternativa al más popular filtro de Kalman. 
Lin y Lin (2000) investigan el comportamiento dinámico y estocástico del coeficiente beta de un factor único en un modelo internacional de activos de capital (ICAPM), y sus consecuencias para los precios de los mercados de valores a nivel de los países y la predicción de rendimientos en los mercados bursátiles mundiales. Lin así como Lin (2000) encuentran que el ICAPM tiene un coeficiente beta estocástico y dinámico, que el mercado mundial de acciones es eficiente e integrado, y el portafolio de mercado mundial es eficiente en el sentido de la relación media-varianza.

\section{Aspectos metodológicos}

El modelo de valuación de activos de capital para México en el que se toma en cuenta no sólo el riesgo sistemático del mercado local sino también el riesgo sistemático que se deriva del funcionamiento del mercado mundial, en el cual además los coeficientes correspondientes, beta doméstica y beta mundial, son variantes en el tiempo se puede representar de la siguiente forma:

$$
\begin{gathered}
r_{i, t}=\alpha_{i}+\beta_{i m, t} r_{m, t}+\beta_{i w, t} r_{w, t}+e_{t} \\
b_{i m, t}=b_{i m, t-1}+h_{t}, \\
b_{i w, t}=b_{i w, t-1}+z_{t} .
\end{gathered}
$$

$r_{i, t}$ es el rendimiento debido a la tenencia del activo $i$-ésimo durante el periodo $t=1, \ldots, T . r_{m, t}$ es el rendimiento del portafolio del mercado doméstico y $r_{w, t}$ corresponde al rendimiento del portafolio mundial de capitales, en ambos casos también durante el periodo $t$. $a_{i}$, o alfa del activo, es un término constante correspondiente al rendimiento autónomo que no se deriva de la exposición al riesgo sistemático total (doméstico y mundial). Por su parte, $b_{i m, t} \mathrm{y} b_{i w, t}$ son, respectivamente, el coeficiente beta doméstico y el coeficiente beta mundial, parámetros que miden la sensibilidad del activo a la volatilidad de los rendimientos del mercado doméstico y la volatilidad del mercado mundial. Por su parte, $e_{t}$ es un término estocástico que da cuenta de la perturbación aleatoria que sufren el rendimiento del activo en el perido $t$. Las dos ecuaciones que aparecen al final del modelo describen la dinámica de caminata aleatoria sin drift que siguen los dos coeficientes beta que se encuentran presentes en la ecuación de los rendimientos del activo $i$-ésimo. $h_{t}$ y $z_{t}$ son perturbaciones aleatorias con media cero y no correlacionadas serialmente. 
En el caso de la estimación de los modelos CAPM y su versión internacional ICAPM con parámetros (betas) constantes, éstos se pueden estimar con métodos convencionales como el de mínimos cuadrados ordinarios y sus variantes como mínimos cuadrados ponderados o la incorporación de matrices ad hoc para obtener estimaciones robustas ante los efectos de heteroscedasticidad y autocorrelación en los residuales. La estimación de un modelo de regresión con parámetros estocásticos se basa en un sistema de ecuaciones diseñadas específicamente para estimar los parámetros cambiantes en el tiempo de acuerdo con un proceso estocástico. De esta forma, el modelo de valuación de activos de capital tomando en cuenta una beta que relaciona los rendimientos del activo con el riesgo sistemático local y otra beta que hace lo mismo pero en relación con el riesgo sistemático internacional se puede estimar mediante el filtro de Kalman (1960), también conocido como de Kalman y Bucy (1961) el cual es un caso especial del modelo de espacio de estados general que se compone de la ecuación de medición y la ecuación de transición.

Un modelo de regresión con parámetros cambiantes se puede escribir entonces como la ecuación de medición:

$$
Y_{t}=X_{t} b_{t}+e_{t}
$$

donde $Y_{t}$ es un vector $T$ x 1 de observaciones de la variable dependiente, en este caso los rendimientos de los activos es una matriz $T$ x $(k+1)$ de observaciones de las $k$ variables independientes o sea los rendimientos del mercado mexicano y los del mercado mundial, $\varepsilon_{t}$ es un vector también $T$ x 1 que contiene perturbaciones aleatorias cuyo valor esperado es igual a cero y cuya varianza es $\sigma^{2}$ que no es aleatoria ni observable. Por su parte, $\beta_{t}$ es un vector $(k+1)$ de estados no observados que evolucionan en el tiempo como un proceso markoviano de primer orden el cual se describe mediante la segunda ecuación del sistema, la ecuación de transición:

$$
b_{t}=F b_{t-1}+h_{t} \quad \text { parat }=1, \ldots, T
$$

El filtro de Kalman estima el estado no observado de las variables $\beta_{t}$ por medio de un procedimiento recursivo utilizando el método de estimadores de máxima verosimilitud (EMV) basado en predicción y actualización. El máximo del logaritmo de la función de verosimilitud está representada por

$$
\operatorname{In} L=-\frac{1}{2} \stackrel{n}{\mathrm{o}} \operatorname{In}\left(2 p f_{t / t-1}\right)-\frac{1}{2} \stackrel{n}{\mathrm{o}} h_{t=1}^{\prime} h_{t / t-1}^{\prime} f_{t / t-1}^{\prime 1} h_{t / t-1}
$$


donde $h_{t \mid t-1}$ es el error de la predicción y $f_{t \mid t-1}$ es la varianza condicional del error de predicción. El error de predicción es la diferencia entre el valor real $E p_{i t}$ y el valor ajustado $E p_{i, t \mid t-1}$ dada la información hasta el periodo $t$-1. Por lo tanto, se tiene.

$$
h_{t / t-1}=E p_{i, t}-E p_{i, t / t-1}
$$

y la varianza condicional del error de predicción es calculada como $f_{t / t-1}=E\left(h_{t / t-1}^{2}\right.$.

Dado que el filtro de Kalman estima toda la serie en una forma bayesiana considerando la nueva información disponible en un mundo de incertidumbre, trae la incertidumbre sobre los estados futuros, así como la incertidumbre sobre el estado actual en el modelo. Adicionalmente, el filtro de Kalman puede capturar la incertidumbre acerca de los estados actuales no observados a través del cambio en la varianza condicional del exceso de rentabilidad de la prima de riesgo de cada activo. La varianza del error de predicción condicional en el filtro de Kalman está dado por

$$
f_{t / t-1}=E p_{i t / t-1} H_{t / t-1} E p_{i t / t-1}^{\dagger}+S_{e}^{2}
$$

donde $H_{t \mid t-1}$ es la matriz de covarianzas de las $b_{i, t}$ condicional en la información hasta el tiempo $t$.

\section{Resultados empíricos}

De la base Economática se tomaron las observaciones de los precios de las 14 acciones cuya clave de pizarra son: ALFAA, BIMBOA, CYDSASAA, COMERCIUBCI, FEMSAUBD, GCARSOA1, KIMBERA, KUOB, PENOLES, SORIANAB, TLEVISACPO, TMMA, VITROA y WALMEXV. El criterio de selección de las acciones fue tomar aquellas acciones que no perteneciendo al sector financiero contasen con el historial de precios más largo, resultando así que el periodo de observaciones que se tomó en cuenta fue desde el dos de diciembre de 1991 hasta el 12 de noviembre de 2013. Como representante del portafolio del mercado local se tomó al IPC de la misma fuente, en tanto que para representar el portafolio del mercado mundial de capitales se tomó el World Capital Market Index calculado y mantenido por Morgan Stanley. Los datos de los activos mexicanos y del mercado local se convirtieron a dólares de los Estados Unidos. Para eliminar la influencia del 
mercado mundial de capitales en la información contenida en el IPC, una vez que se tuvieron los rendimientos medidos en dólares, éstos se regresaron contra el índice del portafolio mundial.

En la tabla 1 se presentan los resultados de las estimaciones del modelo de betas variables en el tiempo que se llevaron a cabo con base en el enfoque sugerido por el filtro Kalman descrito en la sección anterior. Como se puede observar en dicha tabla, la mayor parte de los coeficientes estimados para las ecuaciones del modelo del espacio de estados resultaron significativos en los niveles generalmente aceptados. De acuerdo con el valor del estado final estimado por la técnica del filtro Kalman, se puede observar que hay activos mexicanos que muestran evidencia de responder más que proporcionalmente al riesgo sistemático de carácter doméstico, éste es el caso de ALFAA, BIMBOA, GCARSOA1, KIMBERA y PENOLES cuyas betas estimadas para el estado final son mayores a uno por lo que de acuerdo con el convencionalismo financiero se pueden clasificar como acciones agresivas. Para las betas estimadas de COMERCIUBCI, FEMSAUBD, SORIANAB, TLEVISACPO, VITROA y WALMEXV se tiene que sus valores se encuentran entre $0.5 \mathrm{y}$ por debajo de uno. Únicamente CYDSASAA y KUOB muestran betas locales por abajo del 0.5. En el caso de PENOLES se puede decir que es alta su exposición al riesgo sistemático mundial pues su beta respecto del mercado mundial es mayor a uno. Aunque la beta respecto del mercado mundial que se estimó en el estado final para COMERCIUBCI es también superior a 1, el error estándar es tan alto que vuelve no significativo el parámetro estimado. En los casos de ALFAA, GCARSOA1 y TLEVISACPO se observan también altas exposiciones al riesgo del mercado mundial (betas arriba de 0.9). En casi todos los demás casos se observan betas respecto del mercado mundial con valores arriba de 0.5 , siendo las excepciones CYDSASAA, KUOB y VITROA cuyas betas estimadas se encuentran por debajo de dicho valor.

En varios casos se observan coeficientes alfa no significativos, lo que implica que el riesgo sistemático (local y mundial) está explicado en forma suficiente por el modelo general. En el caso de TMMA no fue posible estimar el modelo especificado pues se presentaron problemas de convergencia, lo que podría deberse a que dicho modelo supone que las betas siguen una caminata aleatoria y ese supuesto no es total o parcialmente congruente con el proceso estocástico que siguen las betas doméstica y mundial que dan cuenta del riesgo sistemático a que está expuesto ese activo o, bien, éstas no cambian. 
El modelo tiene por construcción el factor de riesgo doméstico e internacional ortogonales, ya que el factor doméstico se construyó con los residuos de la regresión del factor doméstico en dólares y el factor internacional, con lo que se evita la colinearidad del factor doméstico e internacional, al ser las variables por construcción independientes. Este resultado de independencia también se observa con las variables instrumentales y los regresores en la regresión en dos etapas. En el caso de que las betas se asuman constantes, para la mayor parte de los activos se observaría autoregresión y heterogeneidad de los residuos a través del tiempo, pero el uso de betas variables construidas por el filtro de Kalman, que permite modelar procesos ARMA (Hamilton, 1994, p. 372), permite considerar la dependencia autoregresiva y a que los rendimientos tengan mayor valor en ciertos momentos durante el tiempo, que no se pueden modelar en modelos de betas constantes.

La figura 1 (veáse anexo) muestra como varían la beta asociada con la rentabilidad residual del índice de precios y cotizaciones de México (beta1F) y la beta asociada con el índice del mercado mundial MSCI (beta2F). Las betas que se presentan en dicha figura fueron pronosticadas utilizando el filtro de Kalman (Bucy) de un periodo hacia delante en el modelo de espacio de estados. El filtro de Kalman permite estimar pronósticos exactos con muestra finita en modelos de coeficientes variantes en el tiempo, es un algoritmo recursivo para que en forma secuencial se actualice el estimado de un paso adelante y la media y varianza del estado dado nueva información.

La figura 1 que muestra los coeficientes beta estimados para cada uno de los activos en la muestra tienen gran variabilidad y su valor depende del periodo de estimación y del activo considerado. En general, la beta local (beta1F) tiene más variación que la beta internacional (beta2F) para la mayoría de los activos. La variabilidad del factor local difiere dependiendo del activo. Activos como KUOB o SORIANAB muestran una variabilidad de la beta con respecto al factor local baja, en cambio activos como COMERCIUBC muestran una variabilidad de la beta del factor local alta. Los demás activos muestran en lo general una variabilidad respecto de la beta doméstica que puede calificarse como intermedia. Se puede decir entonces que el riesgo sistemático de tipo doméstico para la mayoría de los activos mexicanos que se han analizado tiende a ser relativamente estable en el transcurso del tiempo. 


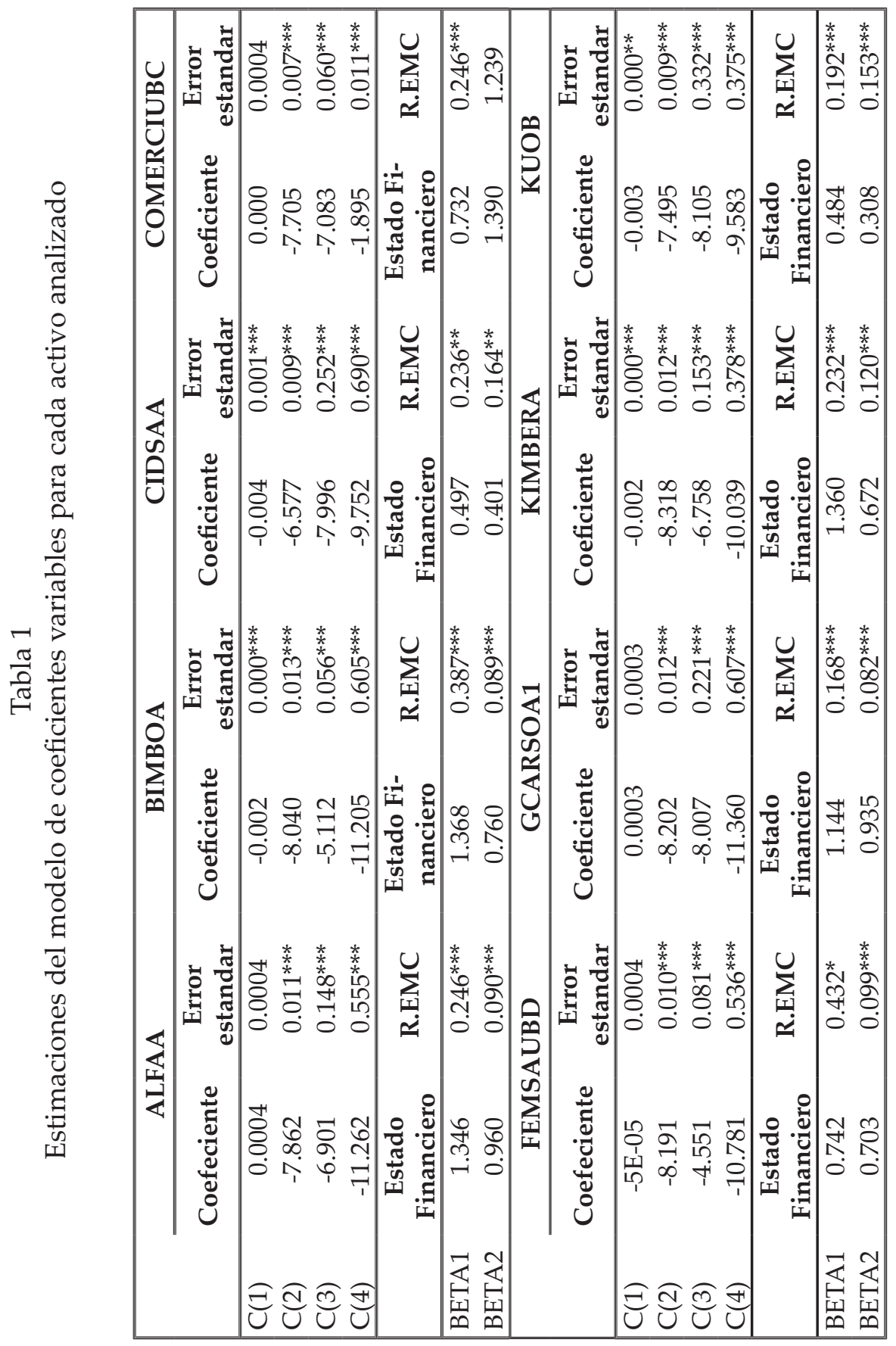




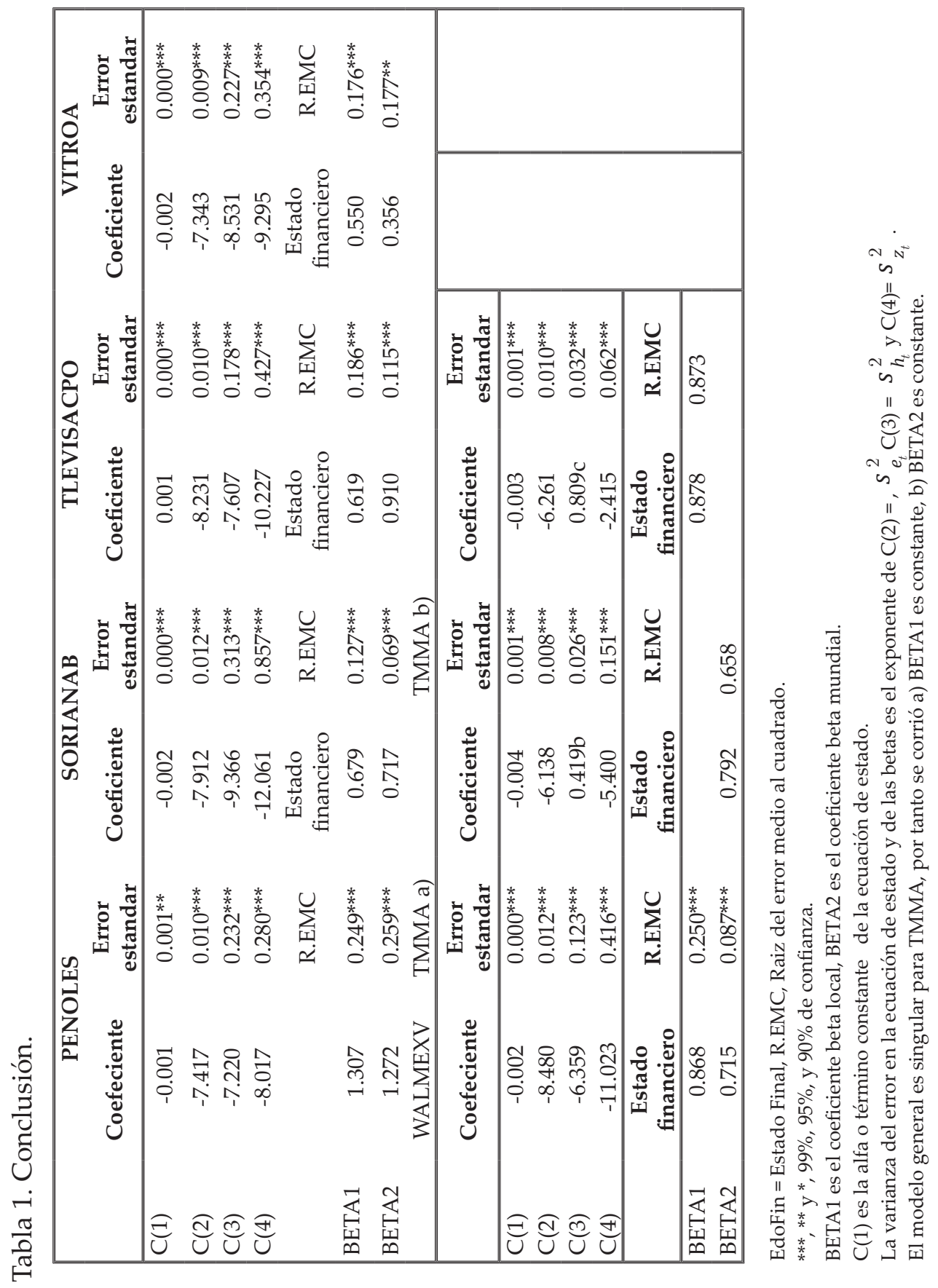


Para la mayoría de los activos, la dependencia del factor internacional, medido por la beta2F, refleja una relativa estabilidad con respecto al riesgo sistemático internacional, aunque para algunos activos como KUOB, VITROA y CIDSAA se observa una disminución del coeficiente beta a partir de 2009, lo que puede deberse a que los efectos de la crisis financiera internacional disminuyeron. Las betas de la acción TMMA no pudieron ser estimadas simultáneamente, por lo que se corrieron estimados asumiendo que uno de los coeficientes betas era constante. Este activo en particular tiene betas con gran volatilidad, tanto las locales como las internacionales.

En términos generales implica que el uso de estimaciones beta constantes pueden causar un grave error cuando la variación en el tiempo de la beta es sustancial. La tendencia central y la volatilidad de las betas variables en el tiempo fluctúan principalmente en perIodos de crisis financiera, como lo fue durante la crisis financiera asiática de 1997, la crisis financiera de Rusia en 1998 y la crisis financiera crediticia mundial de 2008.

La figura 2 (véase anexo) muestra el valor pronosticado para el rendimiento de los activos tomando en cuenta las betas estimadas con el filtro de Kalman (Bucy) a un periodo y la serie original de dichos rendimientos. Se puede observar que la proporción de los rendimientos que es explicada por las betas estimadas difiere con el activo. En activos como CYDSAA, VITROA, o PENOLES, los rendimientos de la serie original fluctúan mucho más y difieren en mayor medida de los rendimientos pronosticados con los coeficientes betas estimados. En cambio, en activos como GCARSOA1 o TELEVISACPO la proporción explicada de la rentabilidad de la serie original por el pronóstico obtenido de los coeficientes betas estimados es mayor. En el caso de TMMA se puede ver que muy poco de la variabilidad de los rendimientos es explicada por el modelo con rendimientos pronosticados, ya sea que varíe el coeficiente beta1 o beta2, lo que es congruente con las estimaciones de la tabla 1.

Los rendimientos pronosticados, en la figura 2, muestran mayor suavidad que los rendimientos originales, lo que muestra que si bien los pronósticos capturan mucha de la variabilidad y cambio de tendencias de los errores, los pronósticos no logran capturar totalmente la variabilidad total que muestra los rendimientos originales. 


\section{Conclusiones}

En este estudio se ha analizado el comportamiento de los rendimientos de un grupo de activos mexicanos utilizando coeficientes beta variantes en el tiempo para capturar el riesgo sistemático local y el riesgo sistemático internacional. Es decir, se ha planteado un modelo internacional de valuación de activos con parámetros variantes en el tiempo para explicar los rendimientos de esos activos. A diferencia de estudios anteriores, el artículo emplea el análisis de series de tiempo para centrarse en el comportamiento de las betas local e internacional y los rendimientos de activos particulares a través del tiempo. El análisis muestra que la estabilidad de la beta es no estacionaria, aunque el movimiento en el tiempo de variación de las betas para algunos activos pudiera fluctuar alrededor de la beta constante que supone el modelo tradicional.

La evidencia presentada en este estudio rechaza que el mercado financiero mexicano esté totalmente integrado al internacional, ya que para la mayoría de los activos considerados es relevante además del factor de riesgo sistemático internacional, un factor de riesgo sistemático local. Los resultados mostrados en páginas previas indican, a diferencia de Lin y Lin (2000), que el mercado mexicano de capitales no está plenamente integrado al mercado internacional de capitales ya que son estadísticamente significativos los coeficientes beta tanto del factor de riesgo internacional como el correspondiente al factor local. Es conveniente destacar también que, en forma similar al estudio de Lin y Lin (2000), nosotros encontramos que las alfas son negativas o no estadísticamente significativas, lo que refuerza el hecho de que considerando coeficientes betas variables el mercado, es condicionalmente eficiente considerando un modelo condicional de valuación de activos de capital.

Las estimaciones de las betas que varían en el tiempo, obtenidas por medio del algoritmo conocido como filtro Kalman, indican que el uso de betas constantes puede causar errores en las estimaciones de la relación entre los rendimientos y las fuentes de riesgo sistemático al que están expuestos los activos del mercado mexicano. Es decir, este estudio muestra la importancia de utilizar modelos de valuación de activos condicionales en el tiempo, en lugar de modelos estáticos, ya que estos últimos pueden resultar en errores de estimación. En el modelo de betas variables locales e internacionales, la mayoría de los activos muestran alfas negativas o no estadísticamente 
significativas, reforzando la hipótesis de eficiencia del mercado financiero cuando se consideran coeficientes beta variables y factores de riesgo sistemático locales e internacionales.

\section{Referencias}

Adler, M. y B. Dumas (1983). "International Portfolio Choice and Corporation Finance: A Synthesis", Journal of Finance, 38 (3). pp. 925-984.

Adrian, Tobias, y Francesco Franzoni (2002). “Learning about beta: An explanation of the value premium", Working paper, MIT.

Alexander, G. J. y N. L. Chervany (1980). “On the Estimation and Stability of Beta”, Journal of Financial and Quantitative Analysis, vol. 15, No. 1, pp. 123-137.

Alford, Alan (1993). "Assessing capital market segmentation: a review of the literature". Stansell, S.R. (edit). International financial market integration. Blackwell. Oxford, UK.

Allen, G.; R. C. M. Impson y I. Karafiath (1994). “An Empirical Investigation of Beta Stability: Portfolio vs. Individual Securities", Journal of Business, Finance and Accounting, vol. 21, No. 6, pp. 909-916.

Avramov. D. y T. Chordia (2006). "Asset Pricing Models and Financial Market Anomalies." Review of Financial Studies, vol. 19 No 3, pp. 1001-1040.

Baesel, J. B. (1974). "On the Assessment of Risk: Some Further Considerations", Journal of Finance, vol. 29, No. 5, pp. 1491-1494.

Barry, C. B. y R. L. Wickler (1976). "Nonstationarity and Portfolio Choice”, Journal of Financial and Quantitative Analysis, vol. 11, No. 2, pp. 217-135.

Bildersee, J. S. y G. S. Roberts (1981). "Beta Instability When Interest Rate Level Changes", Journal of Financial and Quantitative Analysis, vol. 16, No. 3, pp. 375-380.

Blume, M. E. (1971). “On the Assessment of Risk”, Journal of Finance, vol. 26, No. 1, pp. 1-10.

(1979). "Betas and Their Regression Tendencies: Some Further Evidence", Journal of Finance, vol. 34, No. 1, pp. 265-267. 
Brailsford, T. J. y T. Josev (1997). “The Impact of Return Interval on the Estimation of Systematic Risk in Australia", Pacific Basin Finance Journal, vol. 5, No. 3, pp. 357-376.

Braun, P.; D. Nelson y A. Sunier (1995). “Good News. Bad News. Volatility, and Betas." Journal of Finance, vol. 50 pp. 1575-1603.

Carroll, Carolyn y Sears, R. Stephen (1994). Dividend Announcements and Changes in Beta, The Financial Review, vol. 29, No. 3, pp. 371-393.

Choudhry, T. (2002). "The stochastic structure of the time-varying beta: evidence from UK companies", Manchester School, 70 (6), 768-791.

Deb, S. G. y S. Misra (2011). "Are Equity Betas Stable? Evidence from Indian Equity Market". IUP Journal of Applied Finance, vol. 17, No. 4, pp. 5-25.

Errunza, Vihang R. y E. Losq (1985). "International asset pricing under mild segmentation: Theory and test", Journal of Finance, 40 (1). 105-124.

Fabozzi, F. J. y J. C. Francis (1978). "Beta as a Random Coefficient”, Journal of Financial \& Quantitative Analysis, vol. 13, No. 1, pp. 101-116.

Fama, E. y K. French (1992). The Cross-section of expected returns, Journal of Finance, 47, 427-65.

Ferson. W. y C. Harvey (1993). "The Risk and Predictability of International Equity Retums." Review of Financial Studies. 6 pp. 527-566.

Goldberg, M. y A. Vora (1981). "The Inconsistency of the Relationship Between Security and Market Returns", Journal of Economics and Business, vol. 33, No. 2, pp. 97-107.

Grubel, H. G. (1968). "Internationally diversified portfolios", American Economic Review, 58. pp. 1299-1314.

Hamilton, D. James (1994). Time Series Analysis, Princeton University Press, pp. 799.

Hawawini, G. (1983). "Why Beta Shifts as the Return Interval Changes", Financial Analysts Journal, vol. 39, No. 3, pp. 73-77.

Huang, H. (2000). "Tests of regimes-switiching CAPM", Applied Financial Economics, 10, pp. 573-78.

Jostova, G. y A. Philipov (2005). "Bayesian analysis of stochastic betas". Journal of Financial \& Quantitative Analysis, 40(4), pp. 747-778.

Kalman, R. E. (1960). "A new approach to linear filtering and prediction problems", Journal of Basic Engineering, Transactions ASMA, Serie D 82. pp. 35-45.

Kalman, R. E. y R. S. Bucy (1961). "New results in linear filtering and prediction theory", Journal of Basic Engineering, Transactions ASMA, Serie D 83. pp. 95-108.

Ledoil. O.; P. Santa-Clara y M. Wolf (2003). "Flexible Mullivariate GARCH Modeling with an Application to International Stock Markets". Review of Economics and Statistics. 85. pp. 735-747. 
Leusner, J.; J. Akhavein y P. A. V. B. Swamy (1996). “Solving an empirical puzzle in the capital asset pricing model", Finance and Economics Discussion Series, 96-14, Board of Governors of the Federal Reserve System (U.S.).

Levitz, G. D. (1974). “Market Risk and the Management of Institutional Equity Portfolios", Financial Analysts Journal, vol. 30, No. 1, pp. 53-60.

Levy, R. A. (1971). "On the Short Term Stationarity of Beta Coefficients", Financial Analysts Journal, vol. 27, No. 6, pp. 55-62.

Lin, H.-J. y W. T. Lin (2000). A dynamic and stochastic beta and its implications in global capital markets. International Finance, 3 (1), p. 123.

Lintner, J. (1965). "The valuation of risk assets and the selection of risky investments in stock portfolios and capital budgets", Review of Economic and Statistics, 47 (1), pp. 13-37.

Longstaff, F. (1989). "Temporal aggregation and the continuous-time capital asset pricing model", Journal of Finance, 44, pp. 871-87.

López-Herrera, F.; E. Ramírez Cedillo y D. Rodríguez Benavides (2012). “Evidencia de que la Beta de acciones mexicanas cambia en el tiempo", Ortiz. Arango, Francisco y Francisco López. Herrera (Coords). Avances recientes en valuación de activos y administración de riesgos, vol. 3, Universidad Panamericana: México, pp. 175-189.

Markowitz, H. M. (1952). “Portfolio selection”, Journal of Finance, 7 (1), pp. 77-91.

Meyers, S. L. (1973). "The Stationarity Problem in the Use of the Market Model of Security Price Behaviour", Accounting Review, vol. 48, No. 2, pp. 318-322.

Mossin, J. (1966). "Equilibrium in a capital asset market", Econometrica, 34 (4), pp. 768-783.

Ramírez-Cedillo, E.; F. López-Herrera y D. Rodríguez Benavides (2014). “Evidencia adicional sobre la variabilidad de las betas de acciones mexicanas en el tiempo". Martínez-Preece, Marissa; Carlos Zubieta-Badillo, Francisco López-Herrera y Francisco Venegas-Martínez (coord.) Administración de riesgos, vol. V. Universidad Autónoma Metropolitana-Azcapotzalco: México, pp. 173-194.

Schlicht, E. y J. Ludsteck (2006). "Variance estimation in a random coefficients model", Munich Discussion Paper, No. 2006-12, Deparment of Economics, University of Munich.

Sercu, P. (1980). "A generalisation of the International Asset Pricing Model", Revue de l'Association Française de Finance, pp. 91-136.

Shanken. J. (1990). "Inter-temporal Asset Pricing: An Empirical Investigation." Journal of Econometrics. 45, pp. 99-120.

Sharpe. W. F. (1963). "A Simplified Model for Portfolio Analysis", Management Science, vol. 9, No. 2, pp. 277-293. (1970). Portfolio theory and capital markets. McGraw Hill. New York. 
(2000). Portfolio theory and capital markets. McGraw Hill. New York.

Sharpe, W. F. y G. M. Cooper (1972). "Risk Return Classes of New York Stock Exchange Common Stocks, 1931-1967", Financial Analysts Journal, vol. 28, No. 2, pp. 46-54.

Sharpe, W. F.; J. A. Gordon y V. B. Jeffery (1999). Investments, $6^{\underline{a}}$ ed. Prentice Hall. Englewood Cliffs, New Jersey, pp. 176-196.

Solnik, B. (1974). "An equilibrium model of the international capital market", Journal of Economic Theory, 8 (4), pp. 500-524.

Treynor, J. L. (1961). "Market value, time, and risk”, Manuscrito no publicado.

(1962). "Towards a theory of market value of risky assets", Manuscrito no publicado. Se publicó una versión en Korajczyk, Robert A. (ed). 1999. Asset pricing and portfolio performance. Risk Books. London, pp. 15-22. 
Anexo:
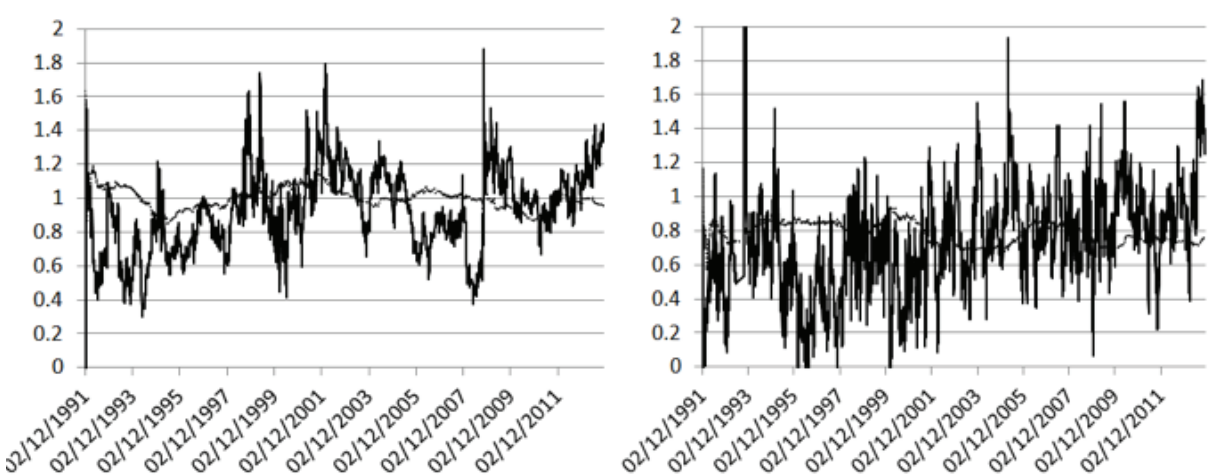

ALFAA

BIMBOA
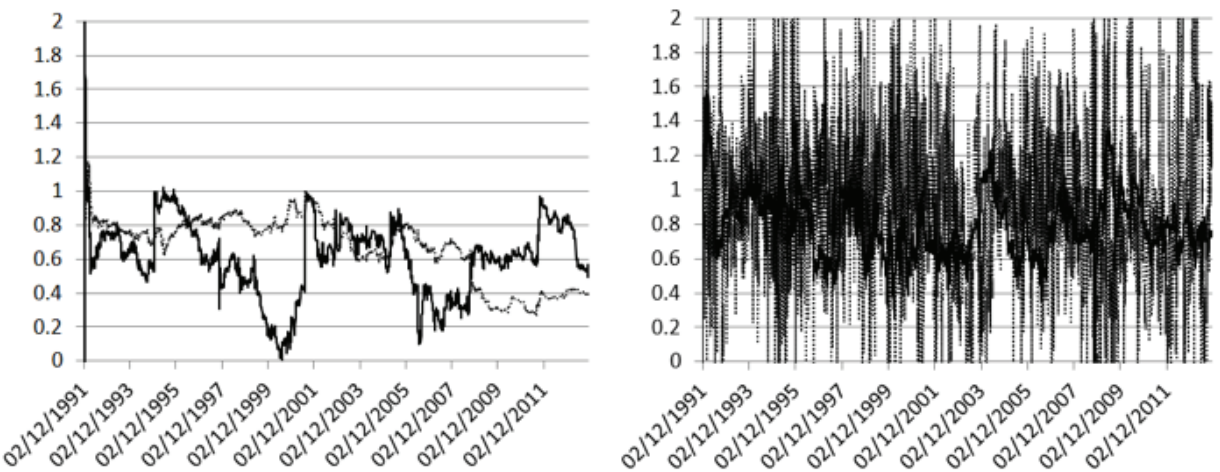

CYDSAA

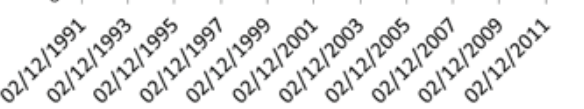

COMERCIUBC

Figura 1

Coeficientes beta variables de cada acción 


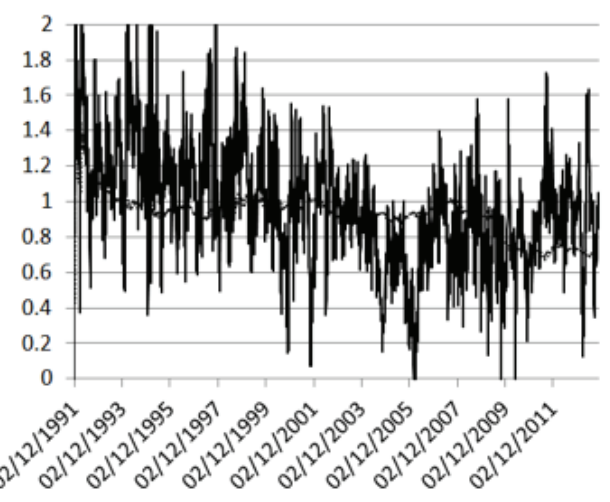

FEMSAUBD
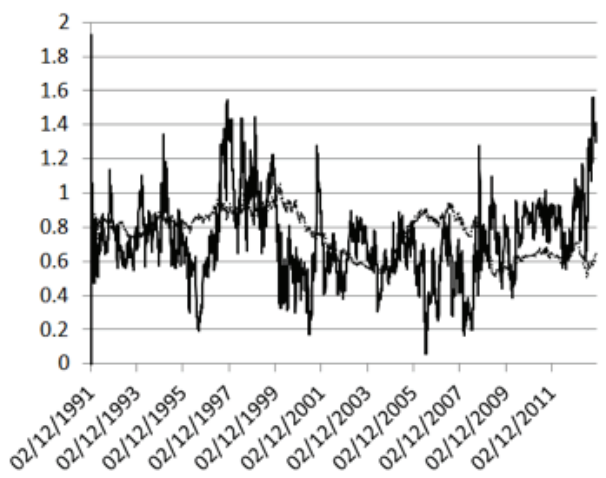

KIMBERA
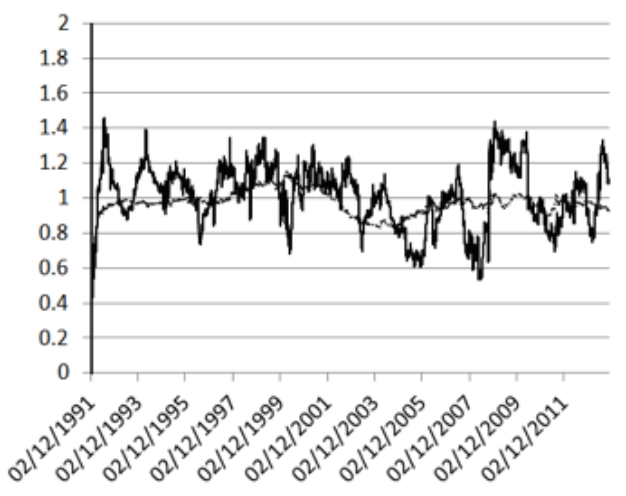

GCARSOA1
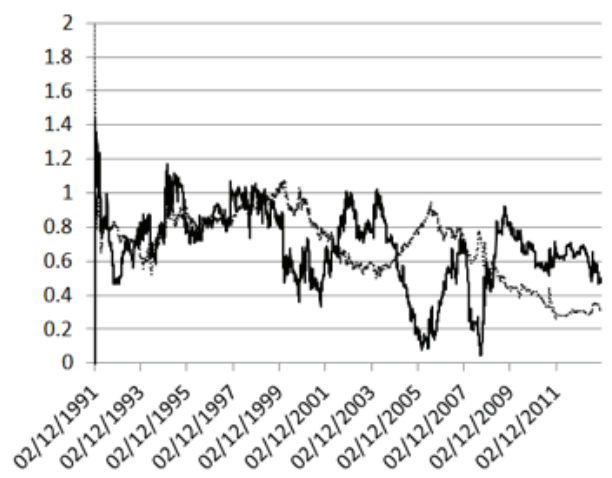

KUOB

BETA 1F

BETA 2F

Figura 1. Continuación. 

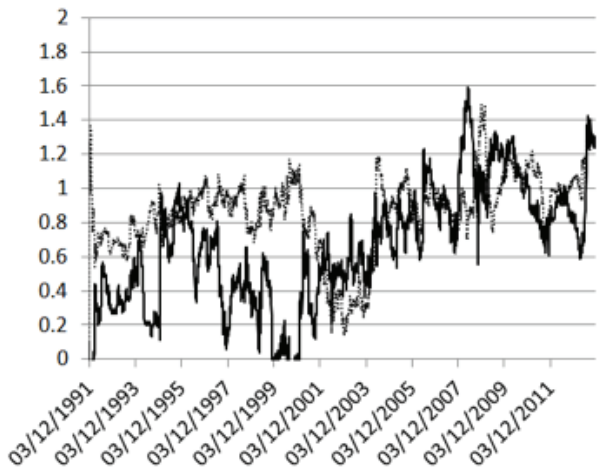

PENOLES
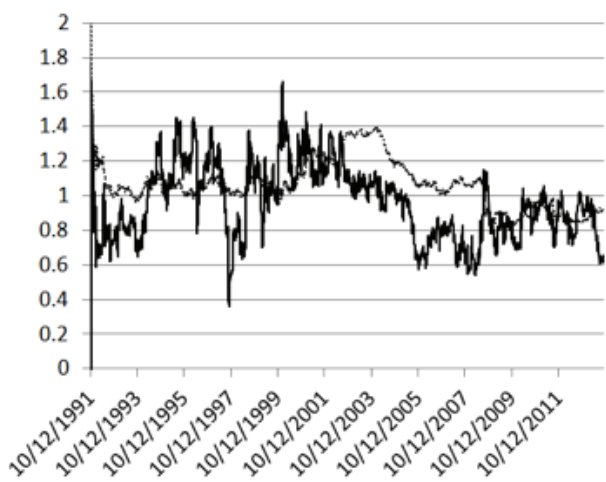

TLEVISACPO
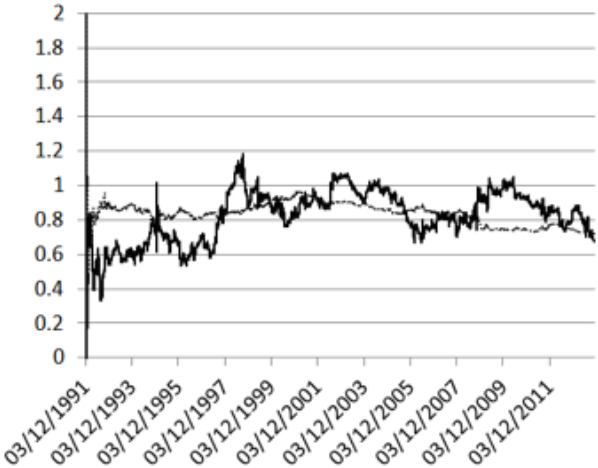

SORIANAB

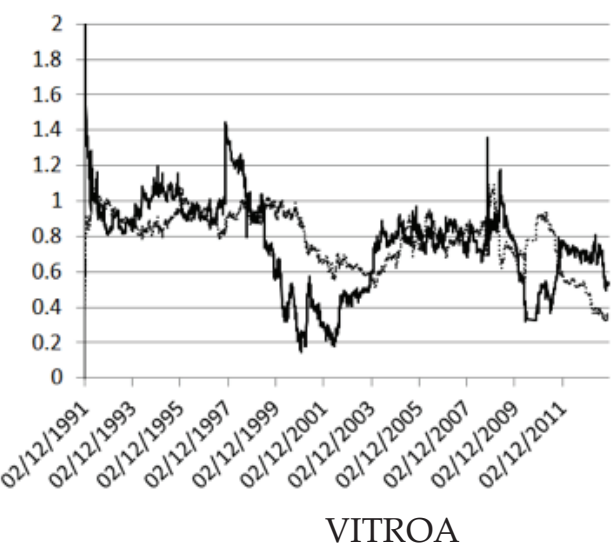

BETA $1 \mathrm{~F}$

BETA 2 F

Figura 1. Continuación. 


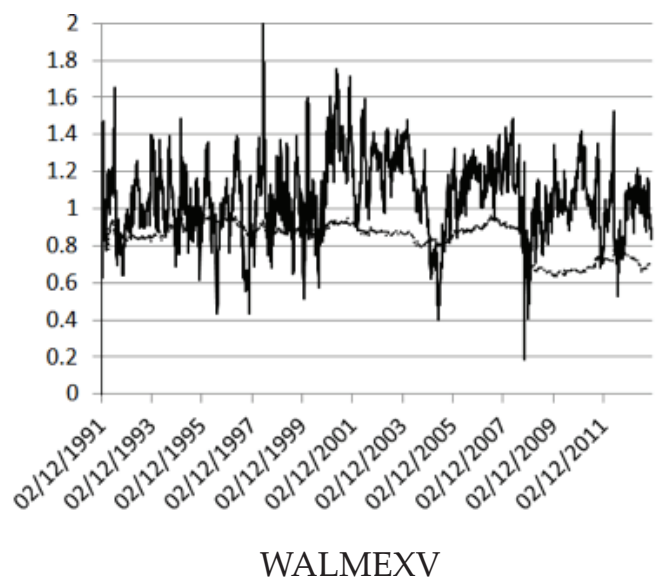

$\longrightarrow$ BETA $1 \mathrm{~F}$

BETA 2F

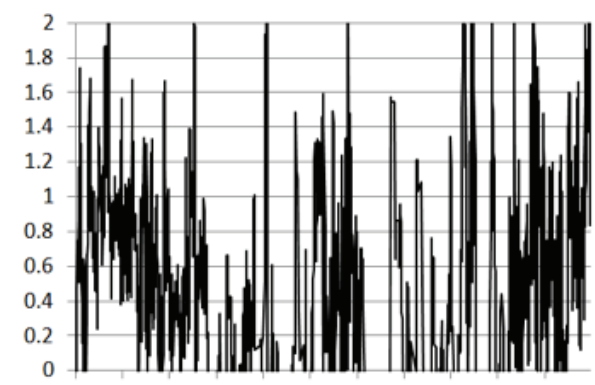

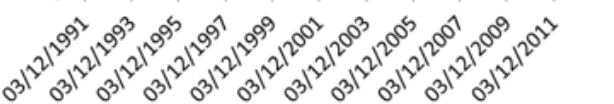

TMMA Beta1, Beta 2 Fija

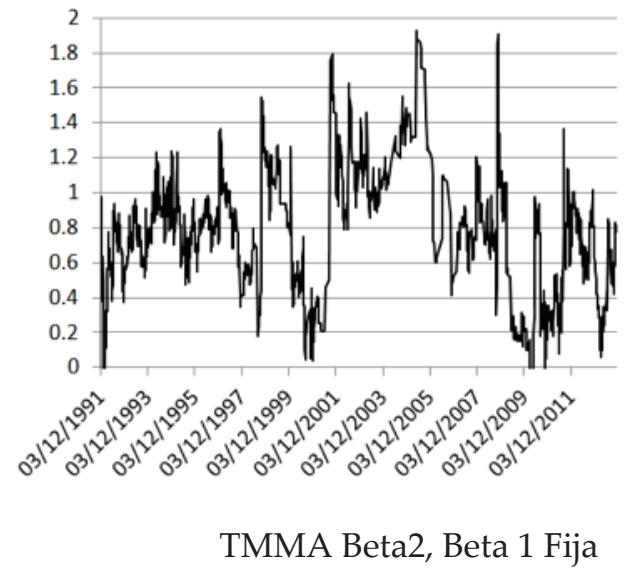

BETA 1F

BETA 2F

Figura 1. Continuación. 

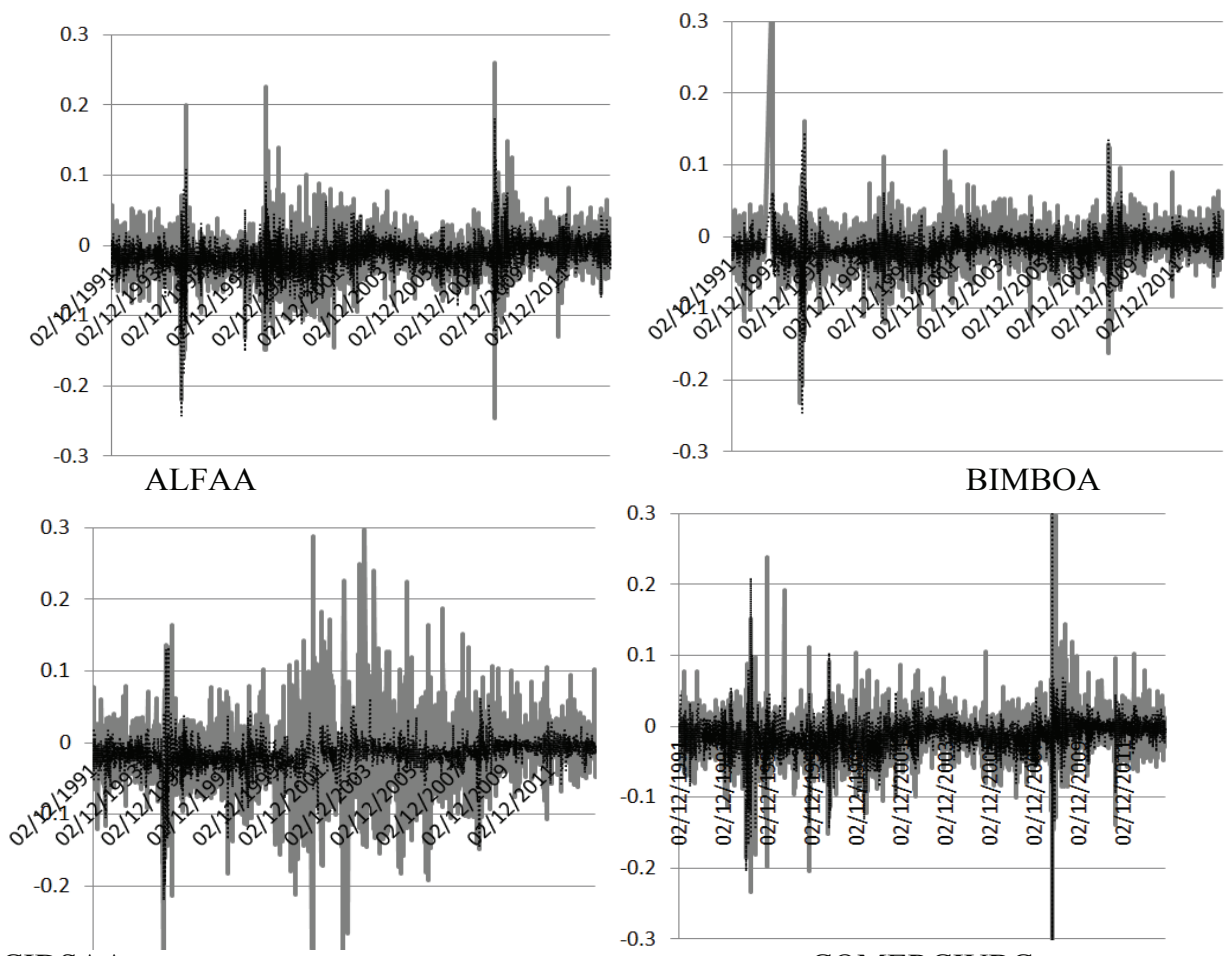

CIDSAA

COMERCIUBC
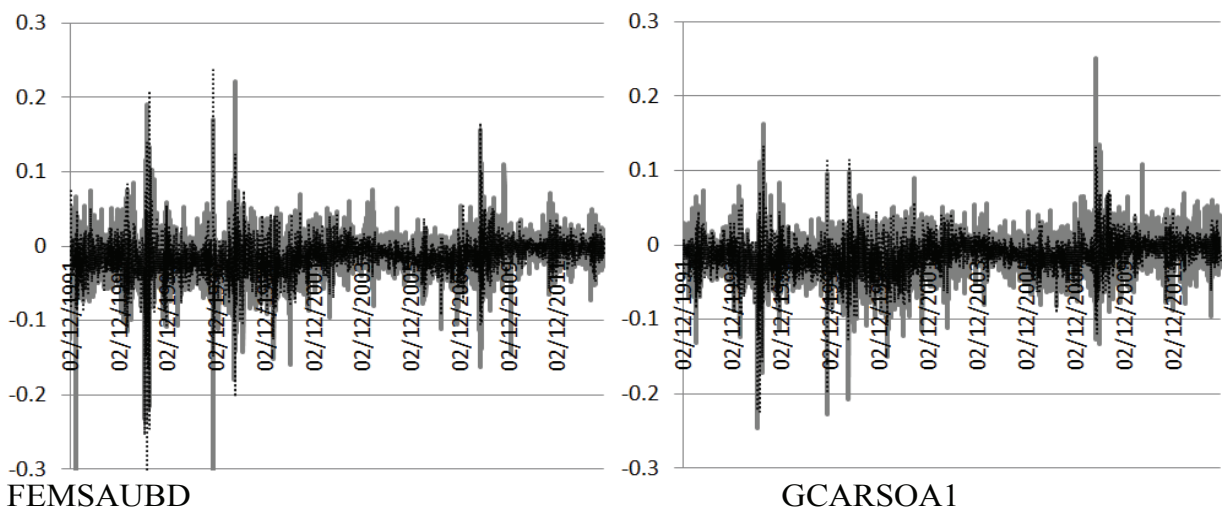

FEMSAUBD

ORIGINAL

PRONÓSTICO

Figura 2

Pronóstico de los rendimientos con base a coeficientes variables beta $v s$ serie original 

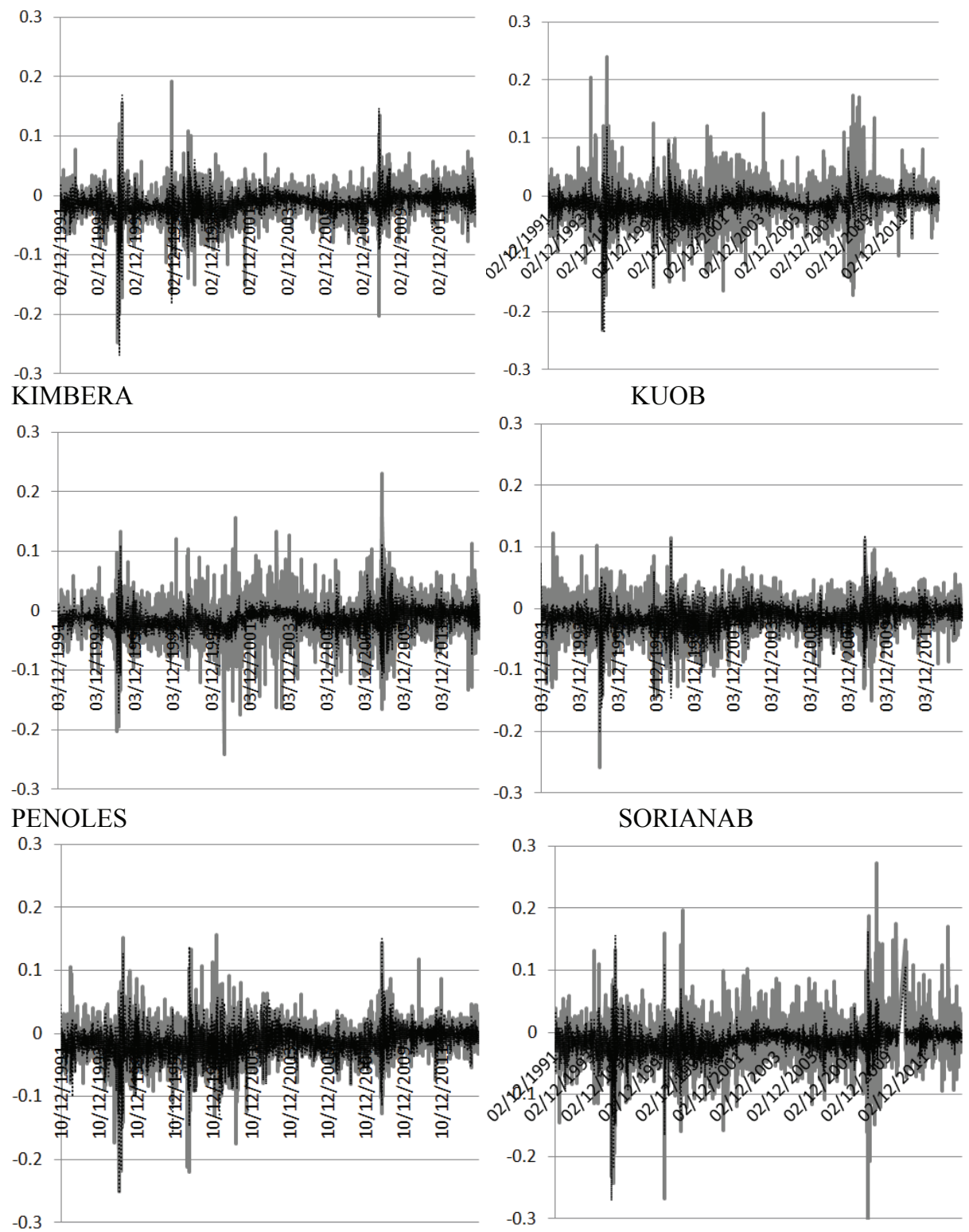

TLEVISACPO

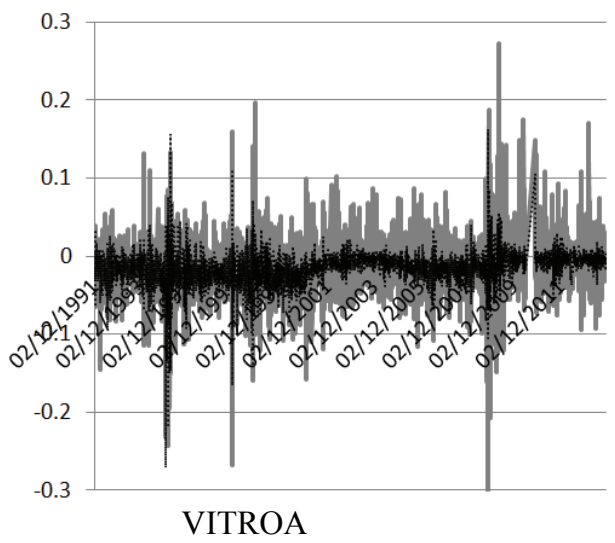

ORIGINAL

PRONÓSTICO

Figura 2. Continuación. 


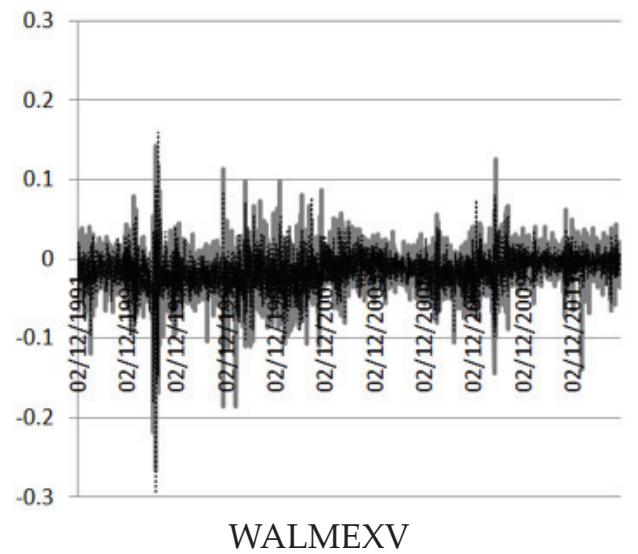

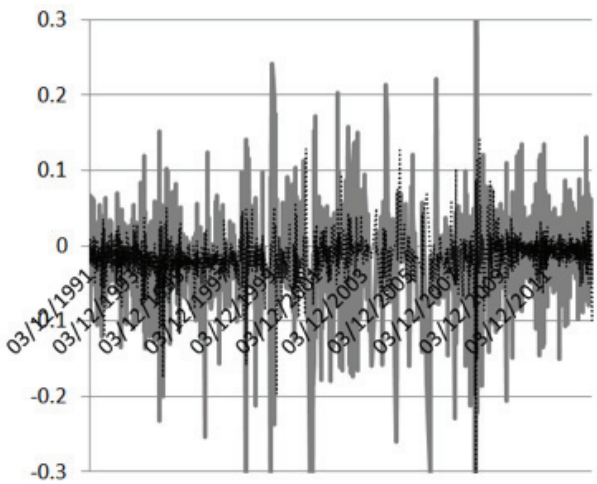

TMMA con BETA1 cambiante, BETA2 fija BETA1 fija

Figura 2. Conclusión

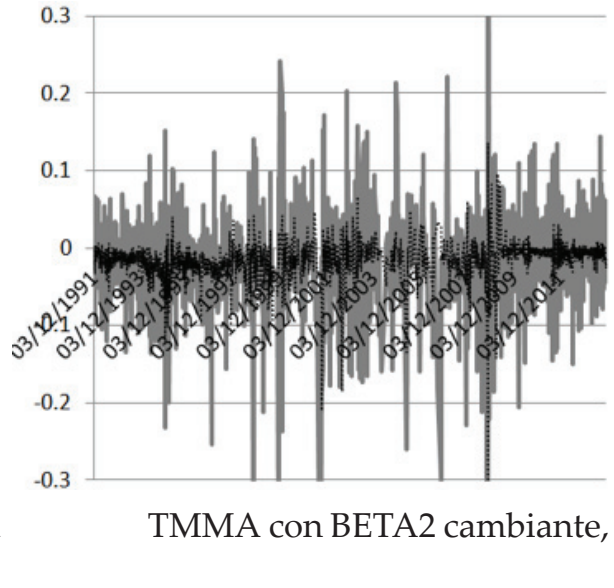

